TECHNICAL RESPONSE

PLANT ECOLOGY

\section{Response to Comment on "Worldwide evidence of a unimodal relationship between productivity and plant species richness"}

\author{
Jason Pither, ${ }^{1 *}$ Lauchlan H. Fraser, ${ }^{2}$ Anke Jentsch, ${ }^{3}$ Marcelo Sternberg, ${ }^{4}$ Martin Zobel, \\ James Cahill, ${ }^{6}$ Carl Beierkuhnlein, ${ }^{7}$ Sándor Bartha, ${ }^{8,9}$ Jonathan A. Bennett, ${ }^{5,6}$ \\ Bazartseren Boldgiv, ${ }^{10}$ Leslie R. Brown, ${ }^{11}$ Marcelo Cabido, ${ }^{12}$ Giandiego Campetella, ${ }^{13}$ \\ Cameron N. Carlyle, ${ }^{14}$ Stefano Chelli, ${ }^{13}$ Anna Mária Csergő, ${ }^{15}$ Sandra Diaz, ${ }^{16}$ \\ Lucas Enrico, ${ }^{16}$ David Ensing, ${ }^{17}$ Alessandra Fidelis, ${ }^{18}$ Heath W. Garris, ${ }^{2}$ \\ Hugh A. L. Henry, ${ }^{19}$ Maria Höhn, ${ }^{20}$ John Klironomos, ${ }^{1}$ Kadri Koorem, ${ }^{5}$ \\ Rachael Lawrence-Lodge, ${ }^{21}$ Peter Manning, ${ }^{22}$ Randall J. Mitchell, ${ }^{23}$ Mari Moora, ${ }^{5}$ \\ Valério D. Pillar, ${ }^{24}$ Gisela C. Stotz ${ }^{6}$ Shu-ichi Sugiyama, ${ }^{25}$ Szilárd Szentes, ${ }^{26}$ \\ Radnaakhand Tungalag, ${ }^{10}$ Sainbileg Undrakhbold, Camilla Wellstein, ${ }^{27}$ Talita Zupo ${ }^{18}$
}

Tredennick et al. criticize one of our statistical analyses and emphasize the low explanatory power of models relating productivity to diversity. These criticisms do not detract from our key findings, including evidence consistent with the unimodal constraint relationship predicted by the humped-back model and evidence of scale sensitivities in the form and strength of the relationship.

I redennick et al. (1), among them many contributors to the original Adler et al. study (2), argue that our findings (3) align closely with those of Adler et al. once their criticisms (described below) are addressed. This is not the case. Tredennick et al. fail to acknowledge key findings of ours that remain at odds with those of Adler et al., including (i) a significantly concave-down, global-extent relationship between productivity and richness; (ii) a significantly concave-down, global-extent quantile regression, consistent with the constraint prediction of the humped-back model (HBM); and (iii) our finding that patterns consistent with the HBM appear more evident when a broad range of productivity is sampled.

Tredennick et al. present three main criticisms of our study: (i) the analyses of the within-site productivity-diversity relationship should have included sample "grid" as a random effect, thereby accounting for our nested sampling design; (ii) our analyses focused too much on the significance of the quadratic term and not enough on the limited explanatory power of the models; and (iii) our figure 2A (3) was "misleading" and should have included a line representing the mixed-effects model for the global-extent relationship. We address each of these in turn.

(i) We agree that including "grid" as a random effect within mixed-effects models would be a reasonable approach. In our within-site analyses, we intentionally replicated the within-site analyses of Adler et al., who did not accommodate the nestedness inherent to their sampling design. In hindsight, we regret not including the results of mixed-effects models for our withinsite analyses in the supplementary materials, as we did for all other analyses. We made our data publicly available, which enabled Tredennick et al. to conduct analyses of their own, finding that $8(29 \%)$ rather than 19 (69\%) of the 28 withinsite analyses yielded a significant concave-down relationship when "grid" is included as a random effect. Crucially, these revised analyses by Tredennick et al. have no effect on the global models we presented that form the main conclusion of the study. Also, thanks to Tredennick et al. making their data and analyses publicly available, we found that the 8 sites that did exhibit a significantly concave-down relationship in their analyses encompassed a significantly larger range of productivity (on the $\log _{10}$ scale) than the 13 sites where no association was found (permutation test on the difference in mean productivity; 9999 permutations; $Z$ score $=2.09 ; P=0.039$ ). Moreover, the probability of detecting a concave-down relationship (i.e., significant quadratic term) over no relationship using the mixed-effects modeling approach tended to increase with increasing biomass range (logistic regression; residual deviance = 22.6 on $19 \mathrm{df} ; P=0.078$ ).

(ii) We recognize that regressions modeling the mean trend between productivity and richness yield limited explanatory power, and stated so in our Report (3). We suggest that Tredennick et al.'s focus on the mean trend is misplaced because, provided one samples a sufficiently broad range of productivity, the HBM predicts a con- straint relationship, whereby richness is constrained to low levels at very low and very high productivity. Our study provided evidence of this, in the form of a significantly concave-down, global-extent quantile regression (both with and without random effects included). Adler et al. also tested for such a constraint relationship (without random effects) but failed to detect it, possibly because of limits to their sampling (3). For our analyses of mean trends, we focused on the form of the relationship, and hence the significance of the quadratic term, because thisnot explanatory power-lies at the heart of the debate surrounding the $\operatorname{HBM}(4,5)$. Our sampling design and sampling scope allowed us to test the sensitivity of the form of the relationship to varying sampling grains and extents.

(iii) We formatted our figure $2 \mathrm{~A}$ (3) with the objective of making it directly comparable to the results presented by Adler et al. (2), specifically their figure 2, and their global-extent regression, which was displayed in their figure 3 . Adler et al. did not account for nested sampling structure in any of their analyses (i.e., using mixed-effects models), including within their global-extent analysis that yielded a significantly concave-down

'Department of Biology, University of British Columbia Okanagan Campus, Kelowna, BC, Canada. ${ }^{2}$ Department of Natural Resource Sciences, Thompson Rivers University, Kamloops, BC, Canada. ${ }^{3}$ Department of Disturbance Ecology, BayCEER, University of Bayreuth, Bayreuth, Germany. ${ }^{4}$ Department of Molecular Biology and Ecology of Plants, Tel Aviv University, Tel-Aviv, Israel. ${ }^{5}$ Department of Botany, Institute of Ecology and Earth Sciences, University of Tartu, Tartu, Estonia. ${ }^{6}$ Department of Biological Sciences, University of Alberta, Edmonton, Alberta, Canada. Department of Biogeography, Bayreuth Center of Ecology and Environmental Research (BayCEER), University of Bayreuth, Bayreuth, Germany. ${ }^{8}$ Hungarian Academy of Sciences Centre for Ecological Research, Institute of Ecology and Botany, Vácrátót, Hungary. ${ }^{9}$ School of Plant Biology, University of Western Australia, Crawley, Australia. ${ }^{10}$ Ecology Group, Department of Biology, National University of Mongolia, Ulaanbaatar, Mongolia. ${ }^{11}$ Department of Environmental Sciences, University of South Africa, Florida, South Africa. ${ }^{12}$ Facultad de Ciencias Exactas, Físicas y Naturales, Universidad Nacional de Córdoba, Córdoba, Argentina. ${ }^{13}$ School of Biosciences and Veterinary Medicine, Plant Diversity and Ecosystems Management Unit, University of Camerino, Camerino, Italy. ${ }^{14}$ Department of Agricultural, Food and Nutritional Science, University of Alberta, Edmonton, Canada. ${ }^{15}$ School of Natural Sciences, Trinity College Dublin, The University of Dublin, Dublin, Ireland. ${ }^{16}$ Instituto Multidisciplinario de Biología Vegetal (IMBIV), National Scientific and Technical Research Council and Facultad de Ciencias Exactas, Físicas y Naturales, Universidad Nacional de Córdoba, Córdoba, Argentina. ${ }^{17}$ Department of Biology, Queen's University, Kingston, Ontario, Canada. ${ }^{18}$ Departamento de Botânica, UNESP Universidade Estadual Paulista, Rio Claro, SP, Brazil. ${ }^{19}$ Department of Biology, University of Western Ontario, London, Ontario, Canada. ${ }^{20}$ Department of Botany, Faculty of Horticultural Science, Corvinus University of Budapest, Hungary. ${ }^{21}$ Department of Botany, University of Otago, Dunedin, New Zealand. ${ }^{22}$ Biodiversity and Climate Research Centre, Senckenberg Gesellschaft für Naturforschung, Germany. ${ }^{23}$ Department of Biology, University of Akron, Akron, OH, USA. ${ }^{24}$ Department of Ecology, Federal University of Rio Grande do Sul, Porto Alegre, Brazil. ${ }^{25}$ Faculty of Agriculture and Life Science, Hirosaki University, Aomori, Japan. ${ }^{26}$ Institute of Plant Production, Szent István University, Gödöllő, Hungary. ${ }^{27}$ Faculty of Science and Technology, Free University of Bozen-Bolzano, Bolzano, Italy. *Corresponding author. E-mail: jason.pither@ubc.ca 
relationship. We therefore opted to show our analogous regression results in figure $2 \mathrm{~A}(3)$. We showed the results of our mixed-effects model for the global relationship in figure S1 (3).

We encourage future research to (i) explore why low species richness (per unit area) is found at the extreme ends of the productivity gradient and (ii) determine the processes that suppress species richness below its potential at intermediate levels of productivity.

\section{REFERENCES}

1. A. T. Tredennick et al., Science 351, 457 (2016) 2. P. B. Adler et al., Science 333, 1750-1753 (2011).
3. L. H. Fraser et al., Science 349, 302-305 (2015).

4. J. P. Grime, J. Environ. Manage. 1, 151-167 (1973).

5. L. H. Fraser, A. Jentsch, M. Sternberg. J. Veg. Sci. 25, 1160-1166 (2014).

16 November 2015; accepted 17 December 2015 10.1126/science.aad8019 


\section{Science MIAAAS}

\begin{abstract}
Response to Comment on "Worldwide evidence of a unimodal relationship between productivity and plant species richness"

Jason Pither, Lauchlan H. Fraser, Anke Jentsch, Marcelo Sternberg, Martin Zobel, James Cahill, Carl Beierkuhnlein, Sándor Bartha, Jonathan A. Bennett, Bazartseren Boldgiv, Leslie R. Brown, Marcelo Cabido, Giandiego Campetella, Cameron N. Carlyle, Stefano Chelli, Anna Mária Csergo, Sandra Diaz, Lucas Enrico, David Ensing, Alessandra Fidelis, Heath W. Garris, Hugh A. L. Henry, Maria Höhn, John Klironomos, Kadri Koorem, Rachael Lawrence-Lodge, Peter Manning, Randall J. Mitchell, Mari Moora, Valério D. Pillar, Gisela C. Stotz, Shu-ichi Sugiyama, Szilárd Szentes, Radnaakhand Tungalag, Sainbileg Undrakhbold, Camilla Wellstein and Talita Zupo (January 28, 2016)

Science 351 (6272), 457. [doi: 10.1126/science.aad8019]
\end{abstract}

Editor's Summary

This copy is for your personal, non-commercial use only.

Article Tools Visit the online version of this article to access the personalization and article tools:

http://science.sciencemag.org/content/351/6272/457.2

Permissions Obtain information about reproducing this article:

http://www.sciencemag.org/about/permissions.dtl

Science (print ISSN 0036-8075; online ISSN 1095-9203) is published weekly, except the last week in December, by the American Association for the Advancement of Science, 1200 New York Avenue NW, Washington, DC 20005. Copyright 2016 by the American Association for the Advancement of Science; all rights reserved. The title Science is a registered trademark of AAAS. 